\title{
HM91 Regimen
}

National Cancer Institute

\section{Source}

National Cancer Institute. HM91 Regimen. NCI Thesaurus. Code C67213.

A regimen administered in four phases that is used for the treatment of disseminated, childhood, anaplastic large cell lymphoma (ALCL). The treatment plan consists of the following 4 phases: COP (cyclophosphamide, vincristine, and prednisone); COPADM (cyclophosphamide, vincristine, prednisone, doxorubicin and methotrexate); VEBBP (vinblastine, etoposide, bleomycin, prednisone); and Sequence 1 (vincristine, methotrexate, cyclophosphamide and doxorubicin). 